\title{
Disability design and innovation in low resource settings: addressing inequality through $\mathrm{HCl}$
}

GIULIA BARBARESCHI

UCL Interaction Centre \& Global Disability Innovation Hub, London, United Kingdom, Giulia.barbareschi.14@ucl.ac.uk

DAFNE ZULEIMA MORGADO RAMIREZ

UCL Interaction Centre \& Global Disability Innovation Hub, London, United Kingdom, d.morgado-

ramirez@ucl.ac.uk

CATHERINE HOLLOWAY

UCL Interaction Centre \& Global Disability Innovation Hub, London, United Kingdom, c.holloway@ucl.ac.uk MANOHAR SWAMINATHAN

Microsoft Research India, Technologies for Emerging Markets, Bangalore, India, Manohar.Swaminathan@microsoft.com

ADITYA VASHISTHA

Cornell University, New York, USA, adityav@cornell.edu

EDWARD CUTRELL

Microsoft Ability Team, Microsoft Research, Redmond, Washington, USA, cutrell@microsoft.com

Approximately $15 \%$ of the world's population has a disability and $80 \%$ live in low resource-settings, often in situations of severe social isolation. Technology is often inaccessible or inappropriately designed, hence unable to fully respond to the needs of people with disabilities living in low resource settings. Also lack of awareness of technology contributes to limited access. This workshop will be a call to arms for researchers in $\mathrm{HCl}$ to engage with people with disabilities in low resourced settings to understand their needs and design technology that is both accessible and culturally appropriate. We will achieve this through sharing of research experiences, and exploration of challenges encountered when planning HCl4D studies featuring participants with disabilities. Thanks to the contributions of all attendees, we will build a roadmap to support researchers aiming to leverage post-colonial and participatory approaches for the development of accessible and empowering technology with truly global ambitions.

CCS CONCEPTS - Human-centered computing Human computer interaction $(\mathrm{HCl}) \cdot \mathrm{HCl}$ Design and evaluation methods $\sim$ Field studies - Social and professional topics $\sim$ User characteristics $\sim$ People with disabilities 
Additional Keywords and Phrases: People with disabilities, Low resourced settings, Accessibility, Cultural context

\section{ACM Reference Format:}

First Author's Name, Initials, and Last Name, Second Author's Name, Initials, and Last Name, and Third Author's Name, Initials, and Last Name. 2018. The Title of the Paper: ACM Conference Proceedings Manuscript Submission Template: This is the subtitle of the paper, this document both explains and embodies the submission format for authors using Word. In Woodstock '18: ACM Symposium on Neural Gaze Detection, June 03-05, 2018, Woodstock, NY. ACM, New York, NY, USA, 10 pages. NOTE: This block will be automatically generated when manuscripts are processed after acceptance.

\section{INTRODUCTION AND BACKGROUND}

People who live in poverty are more likely to live with a disability and people with a disability are more likely to be poor; people who live in low-and middle-income countries are more likely to be poor. This means that of the $10-15 \%$ of people who live with a disability, the majority are less wealthy than their non-disabled peers [23]. although $\mathrm{HCl}$ research and practice has looked at the needs of people with disabilities, most prior work has focused on people with disabilities living in resource-rich settings in North America and Europe. There is a paucity of research that examines the needs of people with disabilities living in the Global South $[8,16]$. In the disability interactions manifesto, Holloway made a call to action that $\mathrm{HCl}$ was ripe to lead substantial change in this area [10]. However, to do so it is clear that the HCl4D, the sub-discipline of ICT4D focusing on the interactions between humans and technology in the context of international development, and disability interaction communities must come together, learn from one another and create a community which can help develop technology and technology-driven interventions which have persons with disability at their core, and ultimately deliver a positive user experience and improve the quality of life of people with disability.

Once a niche field, accessibility and assistive technology have grown to become legal requirements of technology and a flourishing field of research and development within $\mathrm{HCl}$. Example such as the Microsoft $\mathrm{Al}$ for Accessibility fund demonstrate the shift in focus from peripheral to mainstream of inclusive design principles within $\mathrm{HCl}$. In the last decade, researchers and practitioners in the field of $\mathrm{HCl}$ have become increasingly committed to research that is inclusive towards people with disabilities $[4,6,14]$. Much of this work is aimed at ensuring that both current and future technologies are designed and developed according to the abilities [ref], preferences and aspirations of people with disabilities [15, 22]. Increasingly, these studies bake in values such as empowerment, accessibility and inclusion into the methodologies of studies and not only linked to their outputs $[3,5]$.

Similarly, the field of HCI4D has grown significantly in recent years. Linked to the exponential diffusion of mobile phones amongst people living in low- and middle-income countries [9, 24], there has been an increasing number of studies which investigate both how technologies are used and where designs can be improved. Like in the case of accessibility research, $\mathrm{HCI} 4 \mathrm{D}$ and ICT4D strive to understand how technology can be effectively leveraged to deliver meaningful impact to people's lives, ultimately leading to better outcomes for the individual [7]. Moreover, increased sensitivity towards participation and cultural appropriateness through the use of postcolonial approaches promotes research that is co-developed with local communities rather than being predetermined by foreign researchers with different values and often, an external political agenda [1,18].

However, despite these research advances, there are still only a few research studies which look at the intersection of disability and development within the context of $\mathrm{HCl}[16]$. This is concerning, especially as $80 \%$ 
of people with disabilities worldwide live in low resourced settings, are often severely isolated socially, live in multidimensional poverty, inaccessible environments, with limited to no access to assistive technology and facing stigma and discrimination [11]. The few studies which have been conducted have shown: 1) a strong motivation from people with disabilities to interact and take advantage of technology to boost opportunities [19, $21]$; 2) A lack of appropriate, affordable, accessible technology, which further increases the societal gap that leads to marginalization [11]; 3) desire from persons with disability to co-design future technologies [3].

However, there are many challenges that researchers need to overcome when developing and carrying out studies within this space [16]. These include operational and cultural barriers. In a recent review Morgado Ramirez et al. (2020) point out that papers often focus on results and outcomes reporting over accounts of challenges and strategies used to overcome these challenges. Similarly, Ghai et al. 2020, highlight how scholars and practitioners working on research leveraging disability studies in the Gobal South have been largely left out of the assistive technology and accessibility discourse in $\mathrm{HCl}$ [8]. This lack of representation alongside the absence of challenge and best practice reporting go against advice from the HCl4D community, who recommend studies to reflect on methods used (e.g. the use of participatory methods and the need for a multistakeholder approach to tackle complex societal challenges) to help future researchers engage in HCI4D research $[13,17,20]$. The few HCl4D studies carried out with the participation of people with disabilities have shown the potential of $\mathrm{HCl}$ methods and technology, to deliver significant benefits, counteracting some of the physical and societal barriers that are routinely encountered $[2,12,13]$.

Our workshop will bring together the experience of the disability (accessibility, ability-based design, DIX) and development (HCI4D, ICT4D) communities to create and foster a global community driven to overcome the multi-dimensional aspects of poverty faced by people living with disability in low-resource settings. The workshop will represent an avenue for exchange and collaboration, where researchers and practitioners who aim to engage in HCl4D studies involving people with disabilities, will be able to connect with each other and begin to collate experiences. Ultimately, we want these shared research experiences to become the springboard for developing a roadmap to support the execution of a more inclusive global accessibility research agenda in $\mathrm{HCl}$.

\section{LINK TO WEBSITE}

A dedicated space on the Global Disability Innovation Hub website has been created for this workshop and for the development of a global community for global accessibility. The website is available at the following link https://www.disabilityinnovation.com/projects/chi2021

\section{GOALS OF THE WORKSHOP}

The goals of the workshop are the following:

1. Build a community of experts from diverse geographical areas working on two $\mathrm{HCl}$ domains: scholars focusing on research in low resource settings (HCl4D) and scholars working on various aspects of disability (disability studies, accessibility, assistive technology ability-based design, disability interactions). 
2. Understand and maps doubts and challenges encountered when working on $\mathrm{HCl}$ research in low resource settings and with people with disabilities and share best practice of experiences and strategies utilized in successful projects

3. Establish a dedicated space for discussion and continued research at the intersection of disability and development

\section{ORGANIZERS}

Giulia Barbareschi is a Research Fellow in Disability and Assistive Technology at the UCL Interaction Centre and the Global Disability Innovation Hub. Throughout her career Giulia has worked in hospitals, universities and UN agencies in several countries around the world. Giulia is based in London at the UCL Interaction Centre but can often be found in Kenya where she leads the research component of several AT2030 projects on assistive technologies in collaboration with local and international NGOs and start-ups. Her research focuses on understating how current and novel technologies are used to improve the lives of people with disabilities who live in different parts of the world. Giulia's work lies at the intersection of technology, disability and design with strong influences from disability studies and international development.

Dafne Zuleima Morgado Ramirez is Senior Research Associate at the Interaction Centre (UCLIC) of University College London (UCL) and Senior Researcher in Assistive Technology at the Global Disability Innovation Hub. Member of the Global Cooperation on Assistive Technology, founder of the Neurodivergent Staff Network at UCL, Fair Recruitment Specialist at UCL and member of the UCL Disability Equality Steering Group. Zuleima is passionate about multidisciplinary research that aims to achieve technology that is affordable, appropriate, accessible and inclusive. Her research interests are human-computer interaction, assistive and wearable technology, participatory research, social media, social model of disability, adult autism, in development countries and biomedical engineering.

Cathy Holloway is Professor of Interaction Design and Innovation at UCL's Interaction Centre and the Academic Director and co-founder of the Global Disability Innovation Hub (GDI Hub). GDI Hub exists to accelerate disability innovation for a fairer world and Cathy's research revolves around supporting this aim. GDI Hub supports the core values of the Paralympic movement - courage, determination, inspiration and equality and seeks to design our activities in support of the UN Sustainable Development Goals (SDGs).

Manohar Swaminathan (Swami Manohar) is a Principal researcher at Microsoft Research India, where he is part of the Technologies for Emerging Markets group. Manohar is an academic-turned technology entrepreneurturned researcher with a driving passion to build and deploy technology for positive social impact. He has a $\mathrm{PhD}$ in CS from Brown University, was a Professor at the Indian Institute of Science, and has co-founded, managed, advised, and angel-funded several technology startups in India. He has guided over 40 graduate students and has more than 50 refereed publications. His research interests as a professor spanned graphics, virtual reality (taught a graduate course in VR in 1995), and internet technologies. These inform his current research focus, ludic design for accessibility, which puts play and playfulness central to all technology solutions for accessibility, especially in the global south. The current applications include enabling gamers without sight to play mainstream video games, computational thinking for children who are blind, and gaze-tracked interfaces for children with sensory motor impairments.

Aditya Vashistha is an Assistant Professor of Computing and Information Science at Cornell University and a faculty fellow at Cornell Atkinson Center for Sustainability. His research focuses on the design and evaluation 
of technologies that contribute to the socioeconomic development of underserved communities in low-resource environments. This work often spans the domains of healthcare, accessibility, human-computer interaction, and other areas. In addition to designing and building computing systems, he rigorously measures the impact of these systems via large-scale field deployments and mixed-methods evaluations, and actively collaborates with governmental agencies, social enterprises, and grassroots entities. Through these partnerships, his research has transitioned into products that are used by an estimated 220,000 people in Sub-Saharan Africa and on the Indian subcontinent.

Ed Cutrell is a Senior Principal Research Manager at Microsoft Research where he manages the MSR Ability group, exploring computing for disability, accessibility, and inclusive design. Over the years, he has worked on a broad range of $\mathrm{HCl}$ topics, including input tech, visual perception and graphics, intelligent notifications and disruptions, and interfaces for search and personal information management. From 2010-2016, he managed the Technology for Emerging Markets (TEM) group at MSR India, focusing on technologies and systems useful for people living in underserved rural and urban communities in developing countries.

\section{PRE-WORKSHOP PLANS}

The workshop website will be populated with information about the workshop, the call for participation and the participant submission instructions. Participants will be recruited online from the $\mathrm{HCl}$, ICT4D and HCl4D communities, from the research networks of the organizers and on relevant forums and research groups. The organizing committee will work to ensure a diverse range of perspectives is represented at the workshop, and will recruit participants from different geographical areas and working in different domains of accessibility and disability research.

\section{WORKSHOP STRUCTURE}

The workshop will be organized and delivered online, featuring both synchronous and asynchronous components to enable participants to interact with each other in various ways. Furthermore, as the main aim of the workshop is to create a joint space for a new community of researchers working across areas of HCl4D and Disability, we believe that this solution will enable better engagement between participants beyond the length of the virtual workshop.

Ahead of the workshop, the organizers will create a dedicated server of the Discord platform. All the accepted papers will be uploaded to the file repository and dedicated channels will be created to enable people to introduce themselves, share useful resources, highlight gaps and challenges encountered when planning and conducting research on inclusive $\mathrm{HCl} 4 \mathrm{D}$, and discuss shared areas of interest. Workshop contributors will also be asked to send to the organizers a 2-minute accessible video to present the papers they submitted to the workshop. To ensure more time for interactive activities during the synchronous components, participants will be asked to watch the video presentations ahead of the workshop and use the Discord platform to ask questions and engage in discussion.

As we aim for the structure of the workshop to be both accessible and relevant to all participants, we'll create a dedicated channel where will share the proposed agenda below and invite participants to give feedback about the proposed format of the workshop and suggest ways in which activities could be made most accessible to all in consideration of access requirements, technical constraints and bandwidth limitations. 
A key outcome of the workshop is the development of a white paper on "Inclusive HCI4D Research", to be shared with the wider community through the SIGACCESS Newsletter, to help map doubts and challenges encountered when working on $\mathrm{HCl}$ research in low resource settings and with people with disabilities and share useful resources and insights for best practices. Organizers will prepare an initial broad outline that will be uploaded on the Discord server and participants will be invited to provide comment and suggestions on this shared document both ahead and after the workshop.

Synchronous components of the workshop will be carried out using Zoom. To maximise the accessibility of the workshop, support in the form of live closed captioning and live production of detailed meeting notes (using the Otter.ai software) will be provided by the Global Disability Innovation Hub thanks to the funding from the UK FCDO funded AT2030 programme.

The workshop will follow the structure outlined below. To facilitate the exchange and ensure that all participants have a chance to speak and interact, global live discussions involving all participants will be moderated in turn by one of the organizers. Interactive activities, such as challenge mapping or goal setting for the community, will also be moderated by the organizers and will be carried out on Mural, a collaborative digital workspace that features keyboard accessibility. Depending on the number of attendants these brainstorming and collaborative activities might be carried out in a single group or by splitting people across multiple breakout rooms each managed by one organizer. If initial brainstorming is carried out in separate breakout rooms, merging and synthesis across rooms will be led by one representative for each group acting as a spokesperson

\subsection{Workshop Agenda}

The workshop will be held on 16 May JST 2200 - 17 May JST 0200 / 16 May EDT 0900-1300 / 16 May CEST 1500-1900. Below is the proposed agenda for the workshop. Please note that the time 00:00 indicates the starting time of the workshop and is not referred to a particular time of the day in a specific geographical area.

00:00 - 00:10: Introductions from organizers and overview of the structure of the workshop

00:10 - 00:30: "What I wished I would have known" - Video presentation delivered by the organizers in which they share the initial outline of the "Inclusive HCI4D Research" white paper based on their experiences and the contributions made on the Discord platform by participants.

00:30 - 00:40: Moderated Q\&A

00:40 - 01:25: Brainstorming activity using digital post-its to identify main questions and challenges associated with conducting $\mathrm{HCl} 4 \mathrm{D}$ research involving people with disabilities

01:25 - 01:35: Coffee Break \& Informal conversations (for people who wish to remain online).

01:35 - 01:50: Synthesis and merging of identified challenges .

01:50 - 02:20: Interactive activity focused on the organization of challenges that can be encountered at each stage of the research on a temporal roadmap.

02:20 - 02:30: Coffee Break \& Informal conversations (for people who wish to remain online)

02:30 - 03:15: "Where do we go from here?"- Collaborative activity in which participants will discuss the goals of the new community on "Inclusive HCI4D Research", research priorities and best strategies for continued engagement (including the development of a monthly seminar series/reading group).

03:15 - 03:30 Synthesis and merging of suggestions made by participants.

03:30 - 03:40 Coffee Break \& Informal conversations (for people who wish to remain online) 
03:40 - 04:15: General discussion on finalization of plans for the "Inclusive HCI4D Research" white paper including proposed timeline, collaboration platform and potential key editors.

04:15 - 04:30 Workshop wrap-up from the organizers and outline of plans for post-workshop engagement.

If participants consent to it, the workshop will be recorded and a recording with included transcript will be shared amongst attendees after the workshop.

\section{POST-WORKSHOP PLANS}

The aim of this workshop is to bring together researchers working across the fields of HCI4D, ICT4D and accessibility research in order to begin formulating a cohesive vision of methodological approaches and relevant resources to support researchers who aim to make accessibility part of the global agenda in both high and low income countries. The writing of the white paper on "Inclusive HCI4D Research" will be completed according to the timeline and modalities agreed during the workshop and the resulting paper will be published on the website of the workshop and shared with the wider community through the SIGACCESS Newsletter.

Post-workshop discussions will continue on the Discord platform and, based on the suggestions gathered during the workshop, we'll organize a series of monthly seminars and reading group meetings to continue to share experiences of Inclusive HCI4D research, provide engagement opportunities for researcher and practitioners, and grow an active global community that foster collaboration between researchers working in different fields and geographical areas. Finally, to help communicate ongoing work and future priorities to a wider audience, workshop participants will be invited to participate in a series of dedicated blog posts that will be published on the workshop website with the aim of reaching a wider audience and increase awareness around the topic.

\section{CALL FOR PARTICIPATION}

Approximately $80 \%$ of people with disabilities worldwide live in low resourced settings, experiencing severe social isolation, multidimensional poverty, inaccessible environments and disability stigma. More $\mathrm{HCl}$ research that focus on understanding how technology is and could be used to empower people with disabilities who live in low resourced settings is sorely needed, but conducting HCI4D research involving people with disabilities is often challenging especially for researchers who are approaching this field for the first time. I this context, our workshop will focus on identifying challenges, strategies and resources to support scholars who are interested in conducting studies with people with disabilities in low- and middle-income countries. We invite contributions from researchers and practitioners that either

- Describe experiences with strategies to overcome the challenges of performing $\mathrm{HCl}$ research in low resource settings with people with disabilities.

- Discuss challenges associated with the design and development of new $\mathrm{HCl}$ research studies involving people with disabilities living in low resourced settings

- Reflect on the implications of the COVID-19 pandemic in the engagement of people with disabilities in HCI4D research

Submissions can be made in the traditional format of a 4-page paper, but we also welcome submissions in alternative formats, such as photo-journals or videos. Further submission details are available on the workshop's website https://www.disabilityinnovation.com/projects/chi2021. As we hope to both foster 
connections and collaborations among participants while improving the accessibility of the workshop, we'll require participation from at least one author for each submission, but we'll enable participation either in person or virtually.

\section{ACKNOWLEDGMENTS}

Some of the work that has inspired the organization of this workshop has been funded by the UK FCDO (AT2030 Programme 201879-108),UK Global Challenges Research Fund through the Engineering and Physical Sciences Research Council and National Institute for Health Research (EP/R013985/1).

\section{REFERENCES}

[1] Ahmed, S.I. et al. 2015. Residual Mobilities: Infrastructural Displacement and Post-Colonial Computing in Bangladesh. Proceedings of the 33rd Annual ACM Conference on Human Factors in Computing Systems (New York, NY, USA, 2015), $437-446$.

[2] Barbareschi, G. et al. 2020. Bridging the Divide: Exploring the use of digital and physical technology to aid mobility impaired people living in an informal settlement. proceedings of the 22nd International ACM SIGACCESS Conference on Computers and Accessibility (ASSETS '20) (New York, NY, USA, 2020).

[3] Barbareschi, G. et al. 2020. The Social Network: How People with Visual Impairment use Mobile Phones in Kibera, Kenya. Proceedings of the $2020 \mathrm{CHI}$ Conference on Human Factors in Computing Systems (Honolulu, HI, USA, Apr. 2020), 1-15.

[4] Bennett, C.L. et al. 2019. Biographical Prototypes: Reimagining Recognition and Disability in Design. Proceedings of the 2019 on Designing Interactive Systems Conference (San Diego, CA, USA, Jun. 2019), 35-47.

[5] Bennett, C.L. et al. 2019. Making Nonvisually: Lessons from the Field. The 21st International ACM SIGACCESS Conference on Computers and Accessibility (New York, NY, USA, Oct. 2019), 279-285.

[6] Branham, S.M. and Kane, S.K. 2015. Collaborative Accessibility: How Blind and Sighted Companions Co-Create Accessible Home Spaces. Proceedings of the 33rd Annual ACM Conference on Human Factors in Computing Systems (Seoul, Republic of Korea, Apr. 2015), 2373-2382.

[7] Chidziwisano, G.H. et al. 2020. GridAlert: Using a Sensor-Based Technology to Monitor Power Blackouts in Kenyan Homes. Proceedings of the 2020 CHI Conference on Human Factors in Computing Systems (New York, NY, USA, Apr. 2020), 1-13.

[8] Ghai, A. and Reddy, V.Y. Assistive Technology Research and Disability Studies in the Global South: the Need for Synergy.

[9] Ghosh, I. 2016. Contextualizing Intermediated Use in the Developing World: Findings from India \& Ghana. Proceedings of the 2016 CHI Conference on Human Factors in Computing Systems (San Jose, California, USA, May 2016), 355-359.

[10] Holloway, C. 2019. Disability Interaction (DIX): A Manifesto. Interactions. 26, 2 (Feb. 2019), 44-49. DOI:https://doi.org/10.1145/3310322.

[11] Holloway, C. et al. 2018. Scoping Research Report on Assistive Technology. On the road for universal assistive technology coverage. Prepared by the GDI Hub \& partners for the UK Department for International Development. Global Disability Innvoation Hub. (2018).

[12] India, G. et al. 2020. Conceptual Learning through Accessible Play: Project Torino and Computational Thinking for Blind Children in India. Proceedings of the 2020 International Conference on Information and Communication Technologies and Development (New York, NY, USA, Jun. 2020), 1-11.

[13] Jones, J. and Pal, J. 2015. Counteracting dampeners: understanding technology-amplified capabilities of people with disabilities in Sierra Leone. Proceedings of the Seventh International Conference on Information and Communication Technologies and Development (New York, NY, USA, May 2015), 1-10.

[14] Kane, S.K. et al. 2009. Freedom to roam: a study of mobile device adoption and accessibility for people with visual and motor disabilities. Proceedings of the 11th international ACM SIGACCESS conference on Computers and accessibility (Pittsburgh, Pennsylvania, USA, Oct. 2009), 115-122.

[15] Maus, N. et al. 2020. Gaze-guided Magnification for Individuals with Vision Impairments. Extended Abstracts of the 2020 CHI Conference on Human Factors in Computing Systems (New York, NY, USA, Apr. 2020), 1-8.

[16] Morgado Ramirez, D. et al. 2020. Disability design and innovation in computing research in low resource settings. (2020).

[17] Pal, J. et al. 2016. An Accessibility Infrastructure for the Global South. Proceedings of the Eighth International Conference on Information and Communication Technologies and Development (Ann Arbor, MI, USA, Jun. 2016), 1-11.

[18] Rajapakse, R. et al. 2018. Design Artefacts to Support People with a Disability to Build Personal Infrastructures. Proceedings of the 2018 Designing Interactive Systems Conference (Hong Kong, China, Jun. 2018), 277-288.

[19] Vashistha, A. et al. 2014. Educational Content Creation and Sharing by Low-Income Visually Impaired People in India. Proceedings of the Fifth ACM Symposium on Computing for Development (San Jose, California, USA, Dec. 2014), 63-72.

[20] Vashistha, A. et al. 2015. Sangeet Swara: A Community-Moderated Voice Forum in Rural India. Proceedings of the 33rd Annual ACM Conference on Human Factors in Computing Systems (New York, NY, USA, 2015), 417-426.

[21] Vashistha, A. et al. 2015. Social Media Platforms for Low-Income Blind People in India. Proceedings of the 17th International ACM 
SIGACCESS Conference on Computers \& Accessibility (New York, NY, USA, 2015), 259-272.

[22] Wobbrock, J.O. et al. 2011. Ability-Based Design: Concept, Principles and Examples. ACM Transactions on Accessible Computing. 3, 3 (Apr. 2011), 9:1-9:27. DOl:https://doi.org/10.1145/1952383.1952384.

[23] World Health Organization 2011. World Report on Disability.

[24] Wyche, S.P. and Murphy, L.L. 2013. Powering the cellphone revolution: findings from mobile phone charging trials in off-grid Kenya. Proceedings of the SIGCHI Conference on Human Factors in Computing Systems (New York, NY, USA, Apr. 2013), 1959-1968. 